\title{
Comparative Transcriptomic Analysis of Cerebral Organoids and Cortical Neuron Cultures Derived from Human Induced Pluripotent Stem Cells
}

\author{
Annie Kathuria, ${ }^{1-3}$ Kara Lopez-Lengowski, ${ }^{1,2}$ Bradley Watmuff, ${ }^{1-3}$ and Rakesh Karmacharya ${ }^{1-7}$
}

\begin{abstract}
Human induced pluripotent stem cells (iPSCs) can be differentiated along various neuronal lineages to generate two-dimensional neuronal cultures as well as three-dimensional brain organoids. Such iPSC-derived cellular models are being utilized to study the basic biology of human neuronal function and to interrogate the molecular underpinnings of disease biology. The different cellular models generated from iPSCs have varying properties in terms of the diversity and organization of the cells as well as the cellular functions that are present. To understand transcriptomic differences in iPSC-derived monolayer neuronal cultures and three-dimensional brain organoids, we differentiated eight human iPSC lines from healthy control subjects to generate cerebral organoids and cortical neuron monolayer cultures from the same set of iPSC lines. We undertook RNA-seq experiments in these model systems and analyzed the gene expression data to identify genes that are differentially expressed in cerebral organoids and two-dimensional cortical neuron cultures. In cerebral organoids, gene ontology analysis showed enrichment of genes involved in tissue development, response to stimuli, and the interferon- $\gamma$ pathway, while two-dimensional cortical neuron cultures showed enrichment of genes involved in nervous system development and neurogenesis. We also undertook comparative analysis of these gene expression profiles with transcriptomic data from the human fetal prefrontal cortex (PFC). This analysis showed greater overlap of the fetal PFC transcriptome with cerebral organoid gene expression profiles compared to monolayer cortical neuron culture profiles. Our studies delineate the transcriptomic differences between cortical neuron monolayer cultures and three-dimensional cerebral organoids and can help inform the appropriate use of these model systems to address specific scientific questions.
\end{abstract}

Keywords: cerebral organoid, cortical neuron, transcriptomics

\section{Introduction}

ThVestigation of HUMAN neurobiology has historically 1 had significant challenges due to the inability to study live human neuronal tissue. Studies of human brain development have primarily relied on postmortem brain tissue, brain neuroimaging, and extrapolation from animal studies. The ability to reprogram somatic cells to induced pluripotent stem cells (iPSCs) has opened new avenues to culture and grow human neuronal cells ex vivo to study human brain development [1]. Human iPSCs can be differentiated to generate a wide range of neuronal and glial cells, including many cell types relevant to disease biology [2-6]. These techniques utilizing iPSC-derived neuronal cultures are being used widely both to study the neurobiology of brain development as well as to study the cellular processes relevant to the disease biology of neuropsychiatric disorders [7-12]. Recent advances in tissue engineering have also resulted in methods to generate three-dimensional cerebral organoids from human iPSCs $[13,14]$. Cerebral organoids rely on self-organizing abilities of iPSC-derived progenitor cells to recapitulate human cortical development and give rise to many cell types and interconnections found in the human brain [15-18]. These cerebral organoids include mature cortical neuron subtypes and synapses and exhibit functional properties in terms of neuronal firing [19].

We undertook RNA-seq experiments to compare gene expression profiles in cerebral organoids and neuronal

${ }^{1}$ Center for Genomic Medicine, Massachusetts General Hospital, Boston, Massachusetts, USA.

${ }^{2}$ Chemical Biology Program, Broad Institute of MIT \& Harvard, Cambridge, Massachusetts, USA.

${ }^{3}$ Department of Psychiatry, Harvard Medical School, Boston, Massachusetts, USA.

${ }^{4}$ Schizophrenia and Bipolar Disorder Program, McLean Hospital, Belmont, Massachusetts, USA.

${ }^{5}$ Program in Neuroscience and ${ }^{6}$ Program in Chemical Biology, Harvard University, Cambridge, Massachusetts, USA.

${ }^{7}$ Harvard Stem Cell Institute, Cambridge, Massachusetts, USA. 
cultures generated from the same eight human iPSC lines. We analyzed differentially expressed genes (DEGs) in 6-monthold cerebral organoids and 3-month-old two-dimensional cortical neuron cultures. These time points are often used for the study of human pluripotent stem cell-derived models since a diverse array of mature neuronal cells are present at those time points $[20,21]$. Analyses of the transcriptomic data from cerebral organoids and cortical neuron cultures showed relative enrichment of genes involved in tissue development, cellular response, and adhesion in the cerebral organoids, while showing relative enrichment in genes involved in neurogenesis in the monolayer neuron cultures. Gene Set Enrichment Analysis (GSEA) showed that the transcriptome profiles of cerebral organoids had positive correlation to inflammatory signaling, chemical stimulus, and collagen formation, consistent with the gene ontology (GO) analysis. We also compared these transcriptomic profiles with gene expression data in human fetal prefrontal cortex (PFC) at different stages of development and found a greater overlap of the PFC gene expression profiles with the transcriptomic profile from cerebral organoids.

\section{Methods}

\section{Differentiation of iPSCs to two-dimensional cortical neuron cultures}

We used eight human iPSC lines reprogrammed from fibroblasts of healthy control subjects and characterized using standard protocols, with approval from the Massachusetts General Hospital and the McLean Hospital Institutional Review Boards (IRBs), as described previously [22-24]. Cortical neurons were differentiated from human iPSCs that had been cultured and maintained in NutriStem media (Stemgent; 01-0005) using established published protocols [21]. Confluent iPSCs were maintained in N2/B27 medium, comprised half N2 medium, $485 \mathrm{~mL}$ Neurobasal medium (Life Technologies; 21103049), $5 \mathrm{~mL} \mathrm{~N}-2$ supplement (Gibco; 17502001), $5 \mathrm{~mL}$ GlutaMAX (ThermoFisher Scientific; 35050061), $5 \mathrm{~mL}$ penicillin-streptomycin (Gibco; 15140122) and half B-27 medium $10 \mathrm{~mL}$ B-27 supplement (Gibco; 17504044), $480 \mathrm{~mL}$ Dulbecco's modified Eagle's medium (DMEM) (SigmaAldrich; D6421), $5 \mathrm{~mL}$ GlutaMAX (ThermoFisher Scientific; 35050061), $5 \mathrm{~mL}$ penicillin-streptomycin (Gibco; 15140122). On days $1-7$, the medium was supplemented with $10 \mu \mathrm{M}$ SB431542 (Sigma-Aldrich; S4317), $1 \mu \mathrm{M}$ dorsomorphin (Sigma-Aldrich; P5499), and 100 nM LDN193189 (SigmaAldrich; SML0599), with medium replaced daily. On days 829 , the culture was fed daily with the N2/B27 medium. On days 30-90, cells were maintained on BrainPhys neuronal media (StemCell Technologies; 05790) with B-27 supplement, and the medium was changed twice a week.

\section{Generation of three-dimensional cerebral organoids from human iPSCs}

Cerebral organoids were generated with standard published protocols [25] and utilizing STEMdiff ${ }^{\mathrm{TM}}$ Cerebral Organoid Kit (StemCell Technologies; 08570). Human iPSCs were cultured and maintained in NutriStem medium and plated at high density on U-bottom plates to form embryoid bodies (EBs), which were then maintained in EB formation medium for days $1-5$, with medium changed every other day. The EBs were resuspended in induction media on day 5. On Day 7, EBs were embedded in Matrigel and maintained in expansion medium. On day 10 , they were switched to low attachment plates and maintained in expansion medium, with medium changed twice weekly. On day 30 , the medium was supplemented with brain-derived neurotrophic factor with continued medium changes twice weekly as the organoids matured.

\section{RNA extraction}

To obtain RNA, cerebral organoids were added to sterile Eppendorf tubes with $500 \mu \mathrm{L}$ TRIzol (ThermoFisher) and $5 \mathrm{~mm}$ stainless steel beads (Qiagen), which were shaken for 10 min with TissueLyser II (Qiagen). The resulting liquid was transferred to a new Eppendorf tube and $200 \mu \mathrm{L}$ chloroform added. The tube was inverted three times and spun for $20 \mathrm{~min}$ at $14,000 \mathrm{~g}$ at $4^{\circ} \mathrm{C}$. A clear liquid was obtained, which was used in conjunction with the Qiagen RNeasy RNA Mini Kit to obtain RNA samples. RNA extraction from the monolayer cortical neuron cultures was undertaken using standard protocols with the Qiagen RNeasy RNA Mini Kit. List of primers Supplementary Table S5.

\section{Transcriptome analysis}

The Illumina Ribo-Zero TruSeq Stranded Total RNA Library Prep Kit (Illumina) was used to construct the RNA-seq library and the Illumina NovaSeq6000 platform was used for sequencing in the $100 \mathrm{nt}$, paired-end configuration. An average of 60 million reads was obtained for each sample. Trimmed reads with Cutadapt were aligned to the reference genome (hy38 UCSC assembly) for gene expression analyses, using TopHat v2.0.14 and Bowtie v2.10 with default parameters and RefSeq annotation (genome-build GRCh38.p9) [26]. Cufflinks v2.2.1 was used to analyze the distribution of alignments and FPKM (fragments per kilobase of exon model per million reads mapped) values were quantile normalized. Cuffdiff v2.2.1 was used to perform differential expression testing (Supplementary File S1) $[27,28]$. The false discovery rate (FDR) was 0.05 , and sex differences were not considered.

\section{GO \& GSEAS}

GO and KEGG analysis were used on all differentially regulated genes with the Functional Enrichment Analysis unit of HOMER v.3 for process, localization, and molecular function (31). MetaCore+MetaDrug ${ }^{\text {TM }}$ version 19.1 build 69600 was used to analyze metabolic processes. The genes depicted in the figures are the ones that reached significance $(P<0.05)$.

\section{Paraffin embedding}

Cerebral organoids were fixed in 4\% PFA for $30 \mathrm{~min}$, rinsed $3 \times$ in PBS, and dehydrated in a series of ethanol and xylene washes (75\% ethanol: $40 \mathrm{~min}, 80 \%$ ethanol: $40 \mathrm{~min}$, 95\% ethanol: 1:20 min, 100\% ethanol: 25 min, 100\% ethanol: $25 \mathrm{~min}, 100 \%$ ethanol: $30 \mathrm{~min}$, xylene: $30 \mathrm{~min}$, xylene: $30 \mathrm{~min}$, xylene: $1 \mathrm{~h}$, paraffin: $1 \mathrm{~h}$, and paraffin: $1 \mathrm{~h}$ ). The organoids were then embedded in a paraffin block and sectioned on a microtome at $10 \mu \mathrm{m}$ thickness. Sections were placed on a 24 well glass-bottom imaging plate (Greiner Bio-One) and the plate was heated to $60^{\circ} \mathrm{C}$ to adhere the paraffin to the plate. 


\section{Immunohistochemistry}

Sections were rinsed with PBs and PBST (PBS + $0.1 \%$ Triton $\mathrm{X})$ for $10 \mathrm{~min}$ and were subsequently blocked in PBS $+2.5 \%$ goat serum and $2.5 \%$ donkey serum for $1 \mathrm{~h}$. The sections were then stained in primary antibodies diluted in PBS $+1 \%$ goat serum and $1 \%$ donkey serum overnight at $4{ }^{\circ} \mathrm{C}$. The sections were rinsed $3 \times$ in PBS and were then incubated in secondary antibodies diluted in PBS $+1 \%$ goat serum and 1\% donkey serum for $1 \mathrm{~h}$ at room temperature. Slides were rinsed $3 \times$ and Prolong Gold antifade was added. The primary and secondary antibodies used are listed in Supplementary Tables S1 and S2.

A
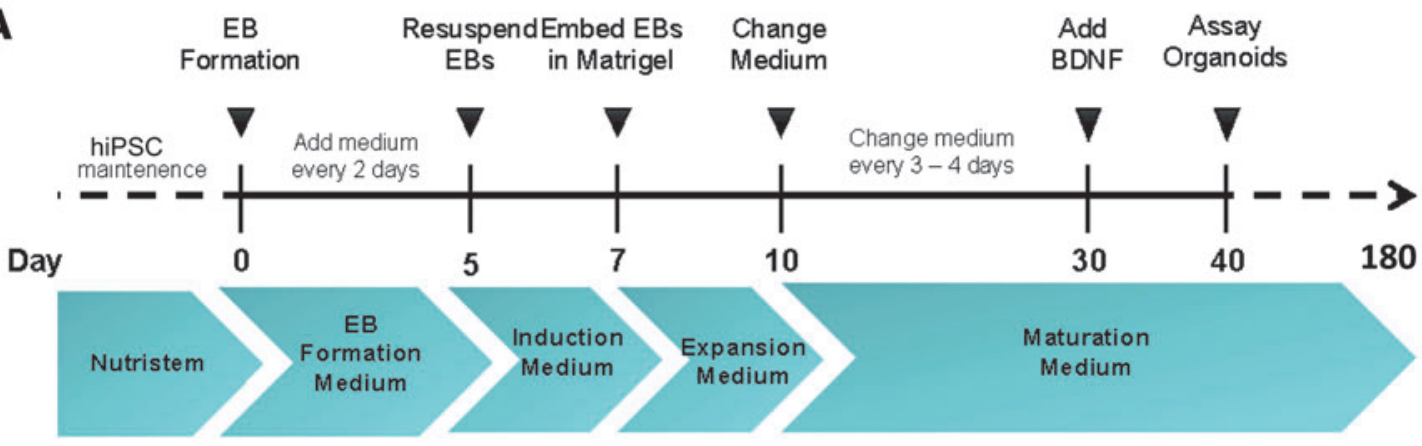

Culture on orbital shaker

B
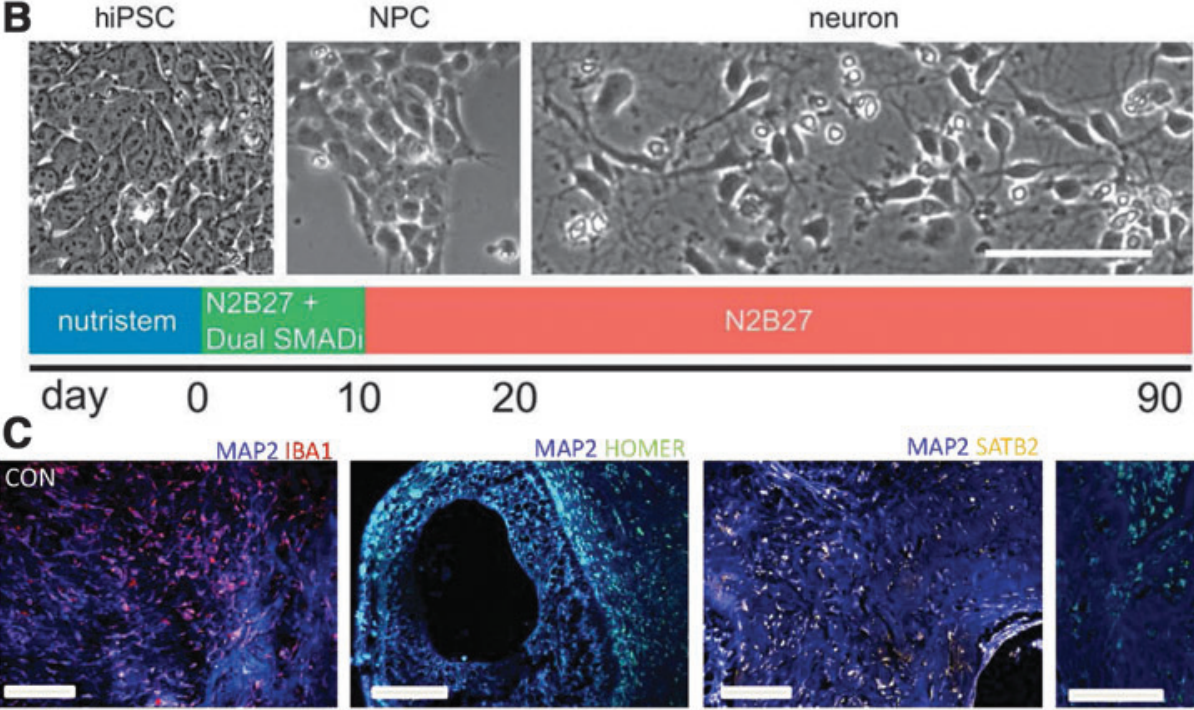

$10 \quad 20$
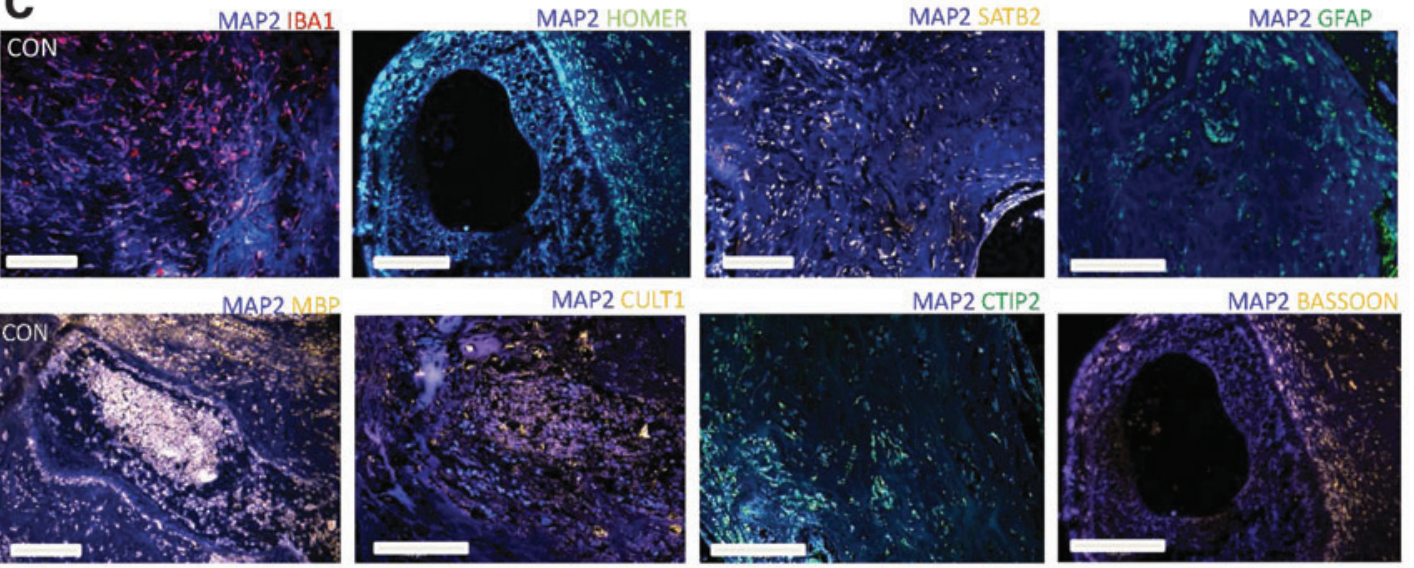

D
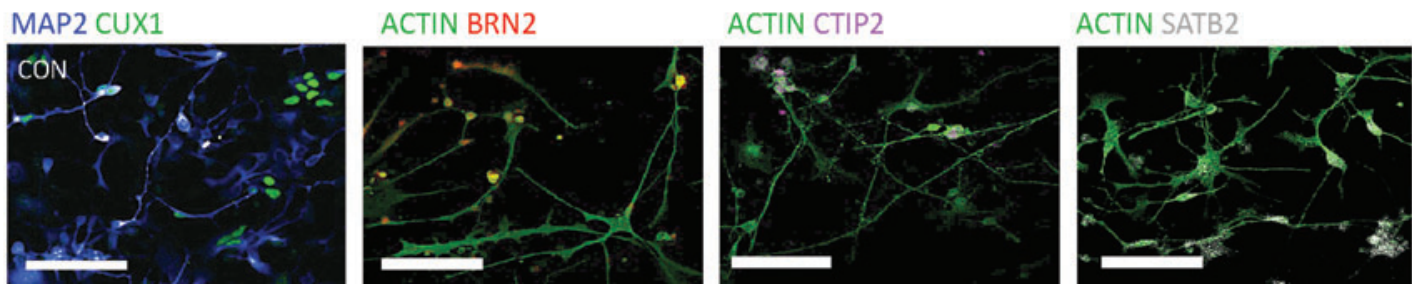

FIG. 1. Three-dimensional cerebral organoids and cortical neurons in monolayer differentiated from human iPSCs. (A, B) Protocols used for generation of cerebral organoids and cortical neuron cultures from human iPSCs. (C) Immunohistochemistry of cerebral organoid slices showing antibody staining for neuronal and glial markers as well as for synaptic proteins. Scale bar: $100 \mu \mathrm{m}$. (D) Immunocytochemistry of monolayer cortical neuron cultures, with antibody staining of markers for different cortical neuron subtypes. Scale bar: $50 \mu \mathrm{m}$. iPSC, induced pluripotent stem cell. 


\section{Immunocytochemistry}

Cells were fixed for $30 \mathrm{~min}$ with $4 \%$ PFA, washed $3 \times$ with PBS, and permeabilized in PBST. The cells were then blocked for $1 \mathrm{~h}$ in PBS $+2.5 \%$ goat serum and $2.5 \%$ donkey serum. The cells were incubated in primary antibodies diluted in PBS $+1 \%$ goat serum and $1 \%$ donkey serum overnight at $4^{\circ} \mathrm{C}$. Following PBS washes, secondary antibodies diluted in PBS $+1 \%$ goat serum and $1 \%$ donkey serum were applied for $1 \mathrm{~h}$ at room temperature. The cells were then rinsed $3 \times$ with PBS and Prolong Gold antifade was added. The primary and secondary antibodies used are listed in Supplementary Tables S1 and S2.

\section{Results}

\section{Generation and characterization of cerebral organoids and cortical neurons}

Eight human iPSC lines reprogrammed from fibroblasts of healthy control subjects, and characterized using standard protocols (Supplementary File S2-S4) as described previously [22-24], were differentiated to generate cerebral organoids and monolayer cortical neuron cultures [21,25]. We characterized the cerebral organoids and cortical neuron cultures with marker analysis, as described previously [22,23]. Both the cerebral organoid tissues and monolayer cortical neuron cultures were positive for a range of neuronal and glial cells, including cortical neurons expressing layer-specific markers such as CUX1, SATB2, and CTIP2 (Fig. 1C, D). All the cerebral organoids showed expression of a range of neuronal and glial cell markers, as we had described previously: MAP2, Ctip2, Satb2, Pax6, TBR2, Cux1, LHX6, glutamine synthetase (GS), GFAP, oligodendrocytes-specific protein/ claudin11 (OSP), myelin basic protein (MBP), and IBA1 (Fig. 1C) [24]. Moreover, when the different cell types were quantified in organoids generated from the different iPSC lines, they showed a consistency in the proportion of cell types in the different organoids (Supplementary Fig. S1) [24]. We undertook total RNA-seq using RNA isolated from the cerebral organoids and the cortical neuron cultures. We generated a heatmap and a volcano plot depicting DEGs, which show distinct differences in gene expression patterns in cerebral organoids generated from the eight iPSC lines when compared to cortical neuron monolayer cultures differentiated from the same iPSC lines (Fig. 2A, B).

\section{GO and GSEA}

We categorized DEGs as enriched in cerebral organoids or enriched in cortical neuron monolayer cultures and rankordered the top 25 hits according to significance ( $P$ value) (Fig. 3A-C). Among the top genes enriched in the cerebral organoids included the cell adhesion protein ameloblastin that is involved in calcium binding [29], the intracellular protein calbindin-2 (calretinin), which is expressed in interneurons and described before in cerebral organoids [30], the calcium sensor copine- 6 that modulates structural plasticity of dendritic spines [31], and the $\mathrm{V}$-set and transmembrane domain containing $2 \mathrm{~A}$ protein that has been found to be expressed neurons and in the cortex www.proteinatlas.org [32]. At the top of the list of genes enriched in monolayer cortical neuron cultures are the calcium-binding protein S100A9, which plays a role in neuron projection development and cellular stress [33], the glycoprotein DMBT1 with roles in calcium-dependent protein binding and signaling pattern recognition receptor activity [34], the epithelial differentiation marker involucrin [35], the calcium-dependent galactose-binding lectin ITLN1 with roles in oxidative
A

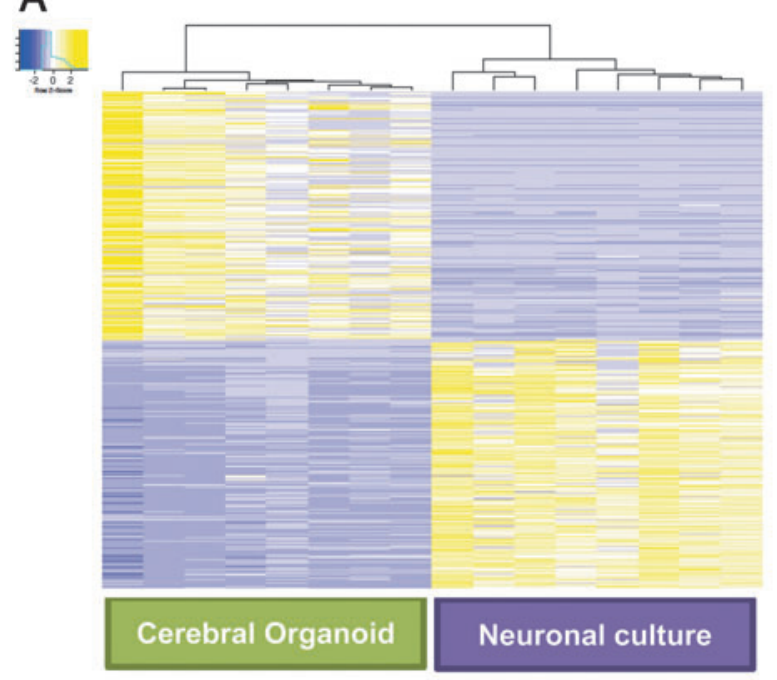

B

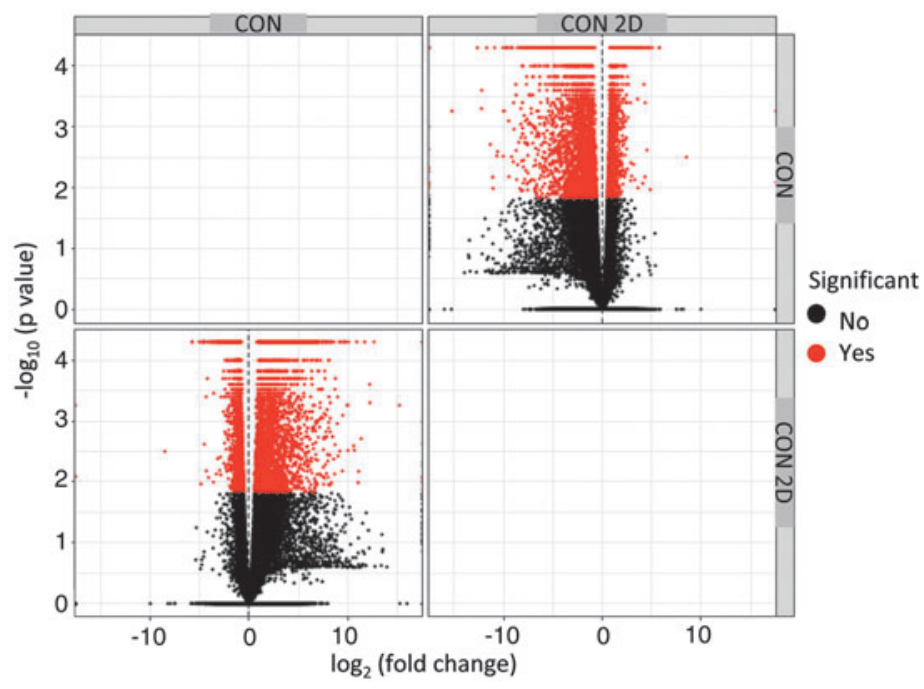

FIG. 2. Gene expression profiles of cerebral organoids and cortical neuron cultures in monolayer differentiated from human iPSCs. (A) Heatmaps of DEGs of 6-month-old cerebral organoids and 3-month-old cortical neuron cultures generated from the same eight human iPSC lines. (B) Volcano plot of gene enrichment in the neuronal cultures and cerebral organoids. DEG, differentially expressed gene. 
A GO Biological Processes

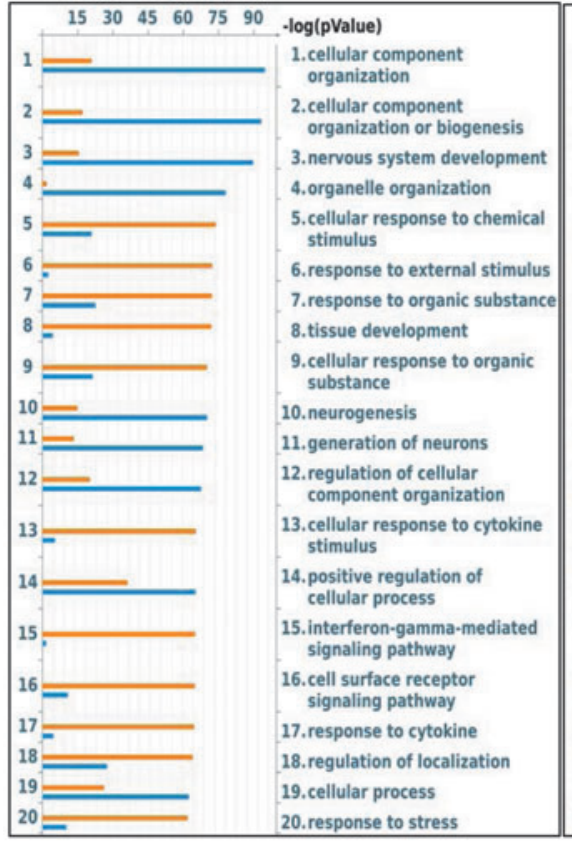

D Enriched in Cerebral organoids

Enriched in Cortical Neurons

HALLMARK database positively corelated HALLMARK_INFLAMMATORY_RESPONSE HALLMARK_INTERFERON_GAMMA_RESPONSE HALLIMARK_ALLOGRAFT_REJECTION HALLMARK_INTERFERON_ALPHA_RESPONSE HALLMARK_IL6_JAK_STAT3_SIGNAUNG HALLMARK database negatively corelated HALLMARK_MYC_TARGETS_V1 HALLMARK_OXIDATIVE_PHOSPHORYLATION HALLMARK_E2F_TARGETS HALLMARK_G2M_CHECKPOINT HALLMARK_DNA_REPAIR HALLMARK_MITOTIC_SPINDLE HALLMARK_MYC_TARGETS_V2 HALLMARK_UNFOLDED_PROTEIN_RESPONSE HALLMARK_UV_RESPONSE_UP HALLMARK_MTORC1_SIGNALING

F REACTOME database positively corelated REACTOME_IMMUNOREGULATORY_INTE REACTOME_COLLAGEN_FORMATION

Reactome data negatively corelated

REACTOME_CEL_CYCLE_MITOTIC

REACTOME_CELL_CYCLE

REACTOME_RESPIRATORY_ELECTRON_TRANSPORT_ATP_SYNTHESIS_BY_CHEMIOSMOTIC_COUPLING_AND_HEAT_PRODUCTION_BY_UNCOUPUNG_PROTEINS REACTOME_PROCESSING_OF_CAPPED_INTRON_CONTAINING_PRE_MRNA

REACTOME_TCA_CYCLE_AND_RESPIRATORY_ELECTRON_TRANSPORT

REACTOME_DNA_REPLICATION

REACTOME_METABOUSM_OF_RNA

REACTOME_MRNA_PROCESSING

REACTOME_AUTODEGRADATION_OF_THE_E3_UBIQUITIN_LIGASE_COP1

REACTOME_TRANSCRIPTION

REACTOME_MRNA_SPUCING

FIG. 3. GO and gene set enrichment analysis of DEGs. (A-C). GO analysis of biological processes, localization, and molecular function for DEGs, rank-ordered according to significance ( $P$ value) for the top 20 hits-depicted as enriched in cerebral organoids (orange) or enriched in cortical neuron cultures (blue). (D-F) GSEA for DEGs in three databases (Hallmark, KEGG, Reactome) tabulated on NES and FDR $q$-value. Positive correlation refers to enrichment in cerebral organoids while negative correlation refers to enrichment in cortical neuron cultures. FDR, false discovery rate; GO, gene ontology; GSEA, gene set enrichment analysis; NES, normalized enrichment score. For ease of reading and color images, the figure can be viewed online.
B GO Localization

C Go Molecular Function

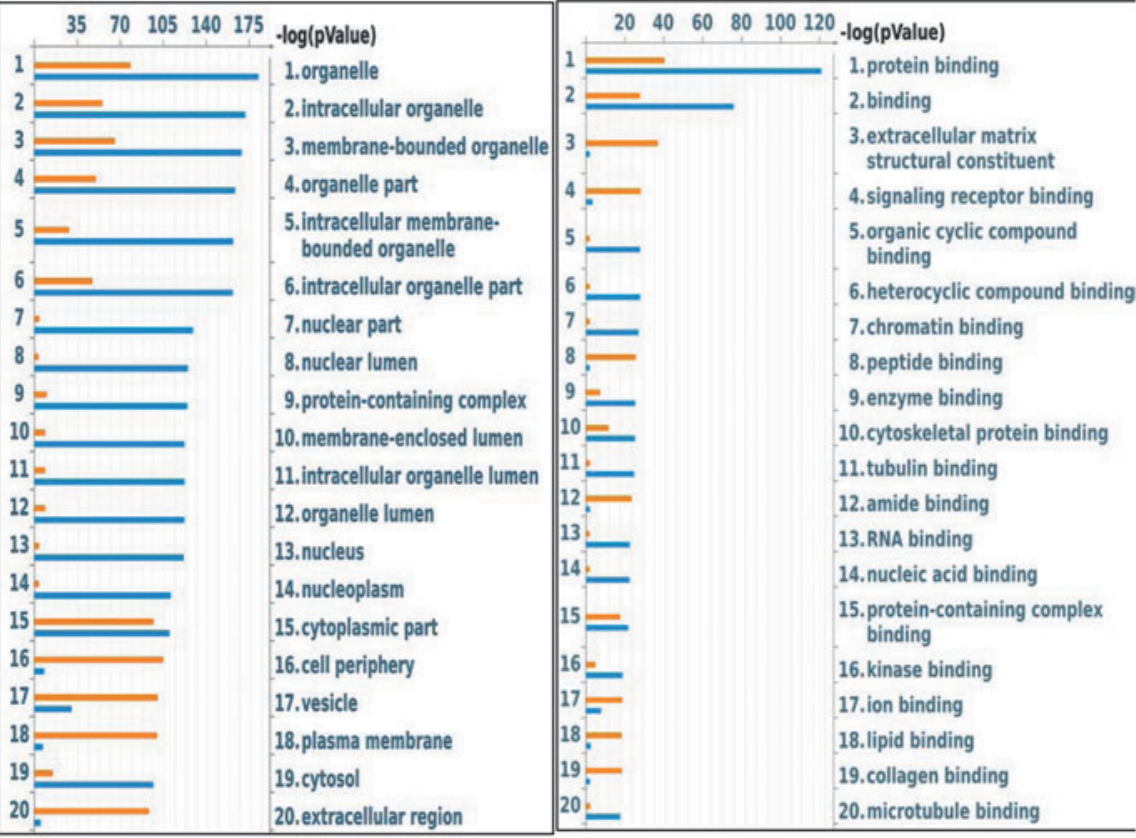

$\mathbf{E}$

KEGG database positively corelated $\quad$ NES FDR q-val

KEGG_INTESTINAL_IMMUNE_NETWORK_FOR_IGA_PRODUCTION 2.3224473

KEGG GRAFT VERSUS HOST DISEASE

KEG__ALOGRAFT_REJECTION

KEGG ASTHMA

KEGG_AUTOIMMUNE_THYROID_DISEASE

\begin{tabular}{ll|l|l|}
1.6169342 & 0.01175785
\end{tabular}

NES

FDR q-val KEGG_RETINOL_METABOLISM

KEGG_TASTE_TRANSDUCTION

KEGG database negatively corelated

KEGG_SPLICEOSOME

KEGG_PROTEASOME

KEGG_OXIDATIVE_PHOSPHORYLATION

KEGG_RIBOSOME

0 KEGG_HUNTINGTONS_DISEASE

0 KEGG_CELL_CYCLE

0 KEGG_ALZHEIMERS_DISEASE

0 KEGG_UBIQUITIN_MEDIATED_PROTEOLYSIS KEGG_PYRIMIDINE_METABOLISM

$\begin{array}{ll}2.2960222 & 0 \\ 2.1917164 & 0\end{array}$

2.1870747

2.1688945

\begin{tabular}{l|l}
2.0843647 & $1.43 \mathrm{E}-04$ \\
\hline
\end{tabular}

\begin{tabular}{ll|l}
1.9698666 & 0.00126499
\end{tabular}

\begin{tabular}{ll|l}
1.8725716 & 0.00348183
\end{tabular}

\begin{tabular}{|l|l}
1.8434963 & 0.00348386
\end{tabular}

\begin{tabular}{ll|l}
1.8066162 & 0.00448561
\end{tabular}

NES FDR q-val

$\begin{array}{rr}-2.8983638 & 0 \\ -2.845986 & 0 \\ -2.8252108 & 0\end{array}$

$-2.8252108 \quad 0$

$\begin{array}{rl}-2.79478 & 0\end{array}$

$-2.7296865 \quad 0$

$-2.7270768$

$-2.66674$

$-2.649579$

$-2.5669615$

$-2.5471928$

NES FDR q-val

$2.2707794 \quad 3.36 \mathrm{E}-04$

\begin{tabular}{ll|l}
2.0252292 & 0.00708604
\end{tabular}

\begin{tabular}{l|l} 
NES & FDR q-val \\
-2.9770489 & 0
\end{tabular}

$-2.9571805$

$-2.956372$

$-2.905678$

$-2.8876874$

$-2.8855407$

2.8836915

$-2.8824213$

$-2.8782897$

$-2.8737936$

$-2.854993$
KEGG_PARKINSONS_DISEASE 
Table 1. Top Ten Differentially Expressed Genes Enriched in Cerebral Organoids and Cortical Neuron Cultures, Listed According to Log-Fold Change

\begin{tabular}{llll}
\hline \multicolumn{2}{c}{$l o g 2($ fold_change $)$} & P_value & \\
\hline Genes enriched in organoids & & & \multicolumn{1}{c}{ Gene description } \\
AMBN & -5.94408 & $5.00 \mathrm{E}-05$ & Ameloblastin \\
CALB2 & -5.41845 & $5.00 \mathrm{E}-05$ & calbindin 2 \\
CPNE6 & -5.16982 & $5.00 \mathrm{E}-05$ & copine 6' \\
VSTM2A-OT1 & -5.02407 & 0.0128 & VSTM2a overlapping transcript 1 \\
VSTM2A & -4.95297 & $5.00 \mathrm{E}-05$ & V-set and transmembrane domain containing 2A \\
C1QL4 & -4.86432 & $5.00 \mathrm{E}-05$ & complement C1q like 4 \\
SLC32A1 & -4.73084 & $5.00 \mathrm{E}-05$ & solute carrier family 32 member 1 \\
VAX1 & -4.68835 & 0.0033 & ventral anterior homeobox 1 \\
SCGN & -4.56377 & $5.00 \mathrm{E}-05$ & secretagogin, EF-hand calcium-binding protein \\
LOC107987397 & -4.50865 & $5.00 \mathrm{E}-05$ & DNA-directed RNA polymerase II subunit RPB1-like \\
Genes enriched in 2D neurons & & \\
S100A9 & 15.3965 & 0.0003 & S100 calcium-binding protein A9 \\
DMBT1 & 12.5401 & $5.00 \mathrm{E}-05$ & deleted in malignant brain tumors 1 \\
IVL & 12.2895 & 0.00025 & Involucrin \\
ITLN1 & 12.1238 & 0.0003 & intelectin 1 \\
MUC14 & 11.5564 & $5.00 \mathrm{E}-05$ & mucin 13, cell surface associated \\
KRT6A & 11.4887 & 0.0109 & keratin 6A \\
PYY & 11.2089 & 0.0013 & peptide YY \\
CDH17 & 10.9958 & 0.00415 & cadherin 17 \\
CLCA1 & 10.8057 & $5.00 \mathrm{E}-05$ & chloride channel accessory 1 \\
TMPRSS4 & 10.6664 & 0.0012 & transmembrane serine protease 4 \\
\hline
\end{tabular}

stress [36], and the transmembrane glycoprotein $M U C 13$ (Table 1) [37]. We performed a GO analysis and found that the most enriched GO processes in cerebral organoids were the interferon- $\gamma$ pathway, tissue development, and cellular response to chemical stimulus. The most significant GO biological processes that were enriched in the monolayer cortical neuron cultures were nervous system development, neurogenesis, and differentiation of neurons (Fig. 3A). GO localization analysis showed relative enrichment in processes involved in vesicle and extracellular region in cerebral organoids and in organelle processes for the monolayer cortical neuron cultures (Fig. 2B). GO molecular function analysis revealed enrichment in the categories of signaling receptor binding and extracellular matrix structural constituent in cerebral organoids and while showing enrichment of the protein binding category in the monolayer cortical neuron cultures (Fig. 3C).

We further carried out GSEA, a computational method that determines whether a set of genes is able to separate two biological categories in a statistically significant manner [38]. We performed GSEA with default parameters in the GSEA software for all expressed genes with FPKM values calculated by Cufflinks against following data sets v6.2: Hallmark, REACTOME, and KEGG. We tabulated positive and negative correlation according to normalized enrichment scores, with positive correlation indicating higher expression in the cerebral organoids and a negative correlation indicating higher expression in the monolayer cortical neuron culture (Fig. 3D-F). Positive correlation was observed with inflammatory response and interferon- $\gamma$ signaling in all three databases (Fig. 3D-F), consistent with pathways that were significant in GO biological processes and localization.
Table 2 lists DEGs enriched in cerebral organoids that are part of the interferon- $\gamma$ signaling pathway, and the expression levels of a number of the key genes were validated by qPCR (Supplementary Fig. S2). Negative correlation indicating higher expression in the monolayer cortical neuron cultures centered on oxidative phosphorylation and cell cycle pathways. Taken together, these results suggest that cerebral organoids have a relatively more robust immune signaling pathways and better response to stimuli.

\section{Comparison of transcriptomic profiles with gene expression in fetal PFC}

The BrainSpan Atlas of the Developing Human Brain contains RNA sequencing data and exon microarray profiles from 16 different brain regions from various stages of human brain development (www.brainspan.org/). We compared transcriptomic profiles in fetal PFC from 8 postconceptional week (pcw) to 5 months after birth with RNA-seq data from the cerebral organoids and the cortical neuron cultures. When gene expression in cerebral organoids and cortical monolayer cultures was compared to the 26,229 genes in the fetal PFC transcriptome at 8-9 pcw, 8226 genes were expressed in cerebral organoids while 4512 were expressed in cortical neurons. At $13-15 \mathrm{pcw}$, of the 27,878 genes expressed in PFC, 9471 were expressed in cerebral organoids and 5485 were expressed in cortical neurons. At $19-24 \mathrm{pcw}, 26,187$ genes were expressed in PFC, out of which 8365 were expressed in cerebral organoids and 4904 expressed in cortical neurons. At postnatal 0-5 months, 25,005 genes were expressed in PFC, out of which 7731 were expressed in cerebral organoids and 5278 expressed in cortical neurons (Fig. 4A).

Significant correlation was observed between the cerebral organoid and PFC transcriptomic profiles (Fig. 4B). The 
Table 2. Differentially Expressed Genes Involved IN THE INTERFERON- $\gamma$ Signaling PathWAY ENRICHED In the Cerebral Organoids (Top 50 Genes AcCording To Log-Fold Change)

\begin{tabular}{|c|c|c|c|}
\hline $\begin{array}{l}\text { DEGs involved } \\
\text { in INF- } \gamma \\
\text { signaling }\end{array}$ & $\begin{array}{c}\log 2 \\
\text { (fold_change) }\end{array}$ & P_value & q_value \\
\hline OAS1 & -6.90769 & 0.001 & 0.00604584 \\
\hline CASP4 & -6.83424 & 0.01125 & 0.0398559 \\
\hline TNFSF10 & -6.77556 & $5.00 \mathrm{E}-05$ & 0.00048351 \\
\hline TNFAIP2 & -6.73353 & $5.00 \mathrm{E}-05$ & 0.00048351 \\
\hline IRF6 & -6.65312 & $5.00 \mathrm{E}-05$ & 0.00048351 \\
\hline PTGS2 & -6.52324 & $5.00 \mathrm{E}-05$ & 0.00048351 \\
\hline$M X 2$ & -6.42068 & 0.0001 & 0.00089722 \\
\hline$X A F 1$ & -6.23497 & $5.00 \mathrm{E}-05$ & 0.00048351 \\
\hline EPSTII & -6.208 & $5.00 \mathrm{E}-05$ & 0.00048351 \\
\hline$O A S 2$ & -5.82765 & 0.00095 & 0.00580028 \\
\hline$C D 86$ & -5.70638 & $5.00 \mathrm{E}-05$ & 0.00048351 \\
\hline$C D 40$ & -5.67515 & $5.00 \mathrm{E}-05$ & 0.00048351 \\
\hline$C S F 2 R B$ & -5.62625 & 0.0049 & 0.0211191 \\
\hline PTAFR & -5.59098 & $5.00 \mathrm{E}-05$ & 0.00048351 \\
\hline HLA-DRBI & -5.588 & 0.01485 & 0.049126 \\
\hline SPII & -5.44754 & 0.00015 & 0.00127748 \\
\hline PTPN6 & -5.26556 & $5.00 \mathrm{E}-05$ & 0.00048351 \\
\hline SP100 & -4.99225 & $5.00 \mathrm{E}-05$ & 0.00048351 \\
\hline SECTM1 & -4.91541 & 0.00245 & 0.0122938 \\
\hline DHX58 & -4.40773 & $5.00 \mathrm{E}-05$ & 0.00048351 \\
\hline$M X 1$ & -4.3122 & $5.00 \mathrm{E}-05$ & 0.00048351 \\
\hline$I L 4 R$ & -4.23393 & 0.0004 & 0.00287947 \\
\hline MYD88 & -4.17387 & $5.00 \mathrm{E}-05$ & 0.00048351 \\
\hline$O A S L$ & -4.11462 & 0.00385 & 0.0174379 \\
\hline HLA-DRB1 & -4.09116 & 0.001 & 0.00604584 \\
\hline CMKLR1 & -4.08248 & 0.0016 & 0.00880692 \\
\hline IFI30 & -3.89748 & $5.00 \mathrm{E}-05$ & 0.00048351 \\
\hline FCGRIA & -3.89359 & 0.0014 & 0.00794668 \\
\hline IL15RA & -3.83344 & $5.00 \mathrm{E}-05$ & 0.00048351 \\
\hline$C D 74$ & -3.83166 & $5.00 \mathrm{E}-05$ & 0.00048351 \\
\hline$P L A 2 G 4 A$ & -3.82071 & 0.00965 & 0.0356166 \\
\hline$O A S 3$ & -3.79986 & $5.00 \mathrm{E}-05$ & 0.00048351 \\
\hline CDHR5 & -3.74691 & $5.00 \mathrm{E}-05$ & 0.00048351 \\
\hline$N M I$ & -3.61544 & $5.00 \mathrm{E}-05$ & 0.00048351 \\
\hline CFH & -3.60805 & 0.00375 & 0.0170959 \\
\hline CDHR5 & -3.6023 & $5.00 \mathrm{E}-05$ & 0.00048351 \\
\hline IL10RA & -3.51981 & 0.0001 & 0.00089722 \\
\hline$F G L 2$ & -3.45642 & $5.00 \mathrm{E}-05$ & 0.00048351 \\
\hline IL6 & -3.40759 & 0.0115 & 0.0405358 \\
\hline CASP 8 & -3.3689 & 0.01025 & 0.037159 \\
\hline PARP12 & -3.20967 & $5.00 \mathrm{E}-05$ & 0.00048351 \\
\hline$G B P 2$ & -3.19186 & $5.00 \mathrm{E}-05$ & 0.00048351 \\
\hline$B A T F 2$ & -3.10439 & $5.00 \mathrm{E}-05$ & 0.00048351 \\
\hline TNFAIP3 & -3.06798 & $5.00 \mathrm{E}-05$ & 0.00048351 \\
\hline$P R K C D$ & -2.80091 & $5.00 \mathrm{E}-05$ & 0.00048351 \\
\hline$I S G 20$ & -2.79392 & $5.00 \mathrm{E}-05$ & 0.00048351 \\
\hline ICAM1 & -2.67642 & $5.00 \mathrm{E}-05$ & 0.00048351 \\
\hline TRIM14 & -2.61415 & $5.00 \mathrm{E}-05$ & 0.00048351 \\
\hline GPR18 & -2.61336 & 0.00325 & 0.0153001 \\
\hline
\end{tabular}

highest correlation between the gene expression profiles was observed between the transcriptomic profile of PFC at 13-15 pcw and the gene expression profile of the cerebral organoids, where $33.9 \%$ of the genes are shared between the two profiles. Given the prominence of the interferon- $\gamma$ pathway in the cerebral organoids in the GO and GSEA analyses, we also examined the expression of these interferon- $\gamma$-related
DEGs in the fetal PFC transcriptomes from the different timepoints, which showed overlap of a large subset of the interferon- $\gamma$-related DEGs in the fetal PFC transcriptomes (Supplementary Fig. S3 and Supplementary Table S3).

We examined specific relevant biological pathways by comparing gene expression patterns in the fetal PFC in relationship to gene expression patterns in cerebral organoids and cortical monolayer cultures by utilizing "nervous system development" and "neurological system process" categories. In the "nervous system development" category, gene expression profiles from the cerebral organoids had higher overall correlation with the transcriptomic profiles in fetal PFC, when compared to gene expression profiles of cortical neuron monolayer cultures (Fig. 4C). At 8-9 pcw, there were 537 nervous system development genes expressed in fetal PFC, out of which 521 were expressed in cerebral organoids while 485 were present in the cortical neuron cultures. At $13-15 \mathrm{pcw}$, there were 465 nervous system development genes expressed in fetal PFC, out of which 441 were expressed in the cerebral organoids while 414 were present in the cortical neuron cultures. At $19-24$ pcw, there were 526 nervous system development genes expressed in fetal PFC, out of which 499 were expressed in cerebral organoids and while 457 were present in the cortical neuron cultures. At $0-5$ months, there were 781 nervous system development genes expressed in fetal PFC, out of which 744 were expressed in the cerebral organoids while 617 were present in the cortical neuron cultures (Fig. 4C).

The "neurological system process" module represents overall development of the nervous system, including formation of synaptic transmission and detection of stimulus. There was a higher correlation between fetal PFC gene expression profiles in this module with cerebral organoid transcriptome profiles, when compared to the cortical neuron cultures (Fig. 4D). Of 999 neurological system process genes expressed in fetal PFC at 8-9 pcw, 935 were expressed in the cerebral organoids while 832 were expressed in the cortical neuron cultures. At 13-15 pcw, there were 955 neurological system process genes expressed in fetal PFC, of which 888 were expressed in the cerebral organoids while 781 were expressed in the cortical neuron cultures. Similar results were seen at other fetal PFC development timepoints, including at 19-24 pcw and at 0-5 months, with a higher correlation between the PFC gene expression profiles with gene expression profile of cerebral organoids compared to that of cortical neuron monolayer cultures (Fig. 4E, F). These results show that the cerebral organoid gene expression profile, compared to the cortical neuron culture profile, tracks more closely with PFC gene expression profiles from the prenatal stage and the early postnatal period, while less overlap in gene expression occurs in the cortical neuron cultures. Moreover, the correlation of gene expression patterns in the cerebral organoids with the PFC transcriptome increased with the age of fetal PFC samples (Fig. 4A-F).

\section{Discussion}

The suitability of specific iPSC-derived ex vivo models need to be guided by the nature of the scientific questions being asked [39]. Many stem cell-based studies have used iPSC-derived neural progenitor cells or differentiated 

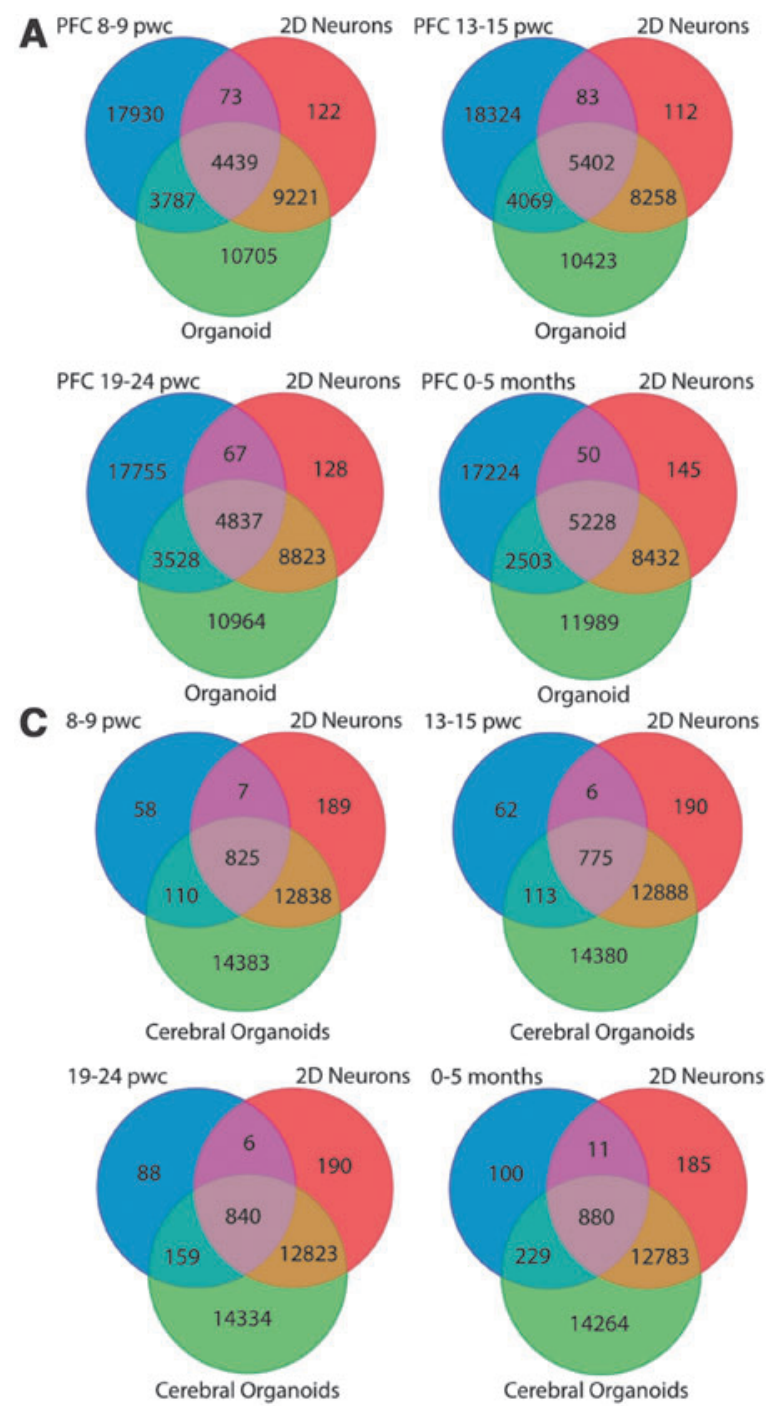

E Correlation Nervous system development
B

\section{Correlation of full transciptome}
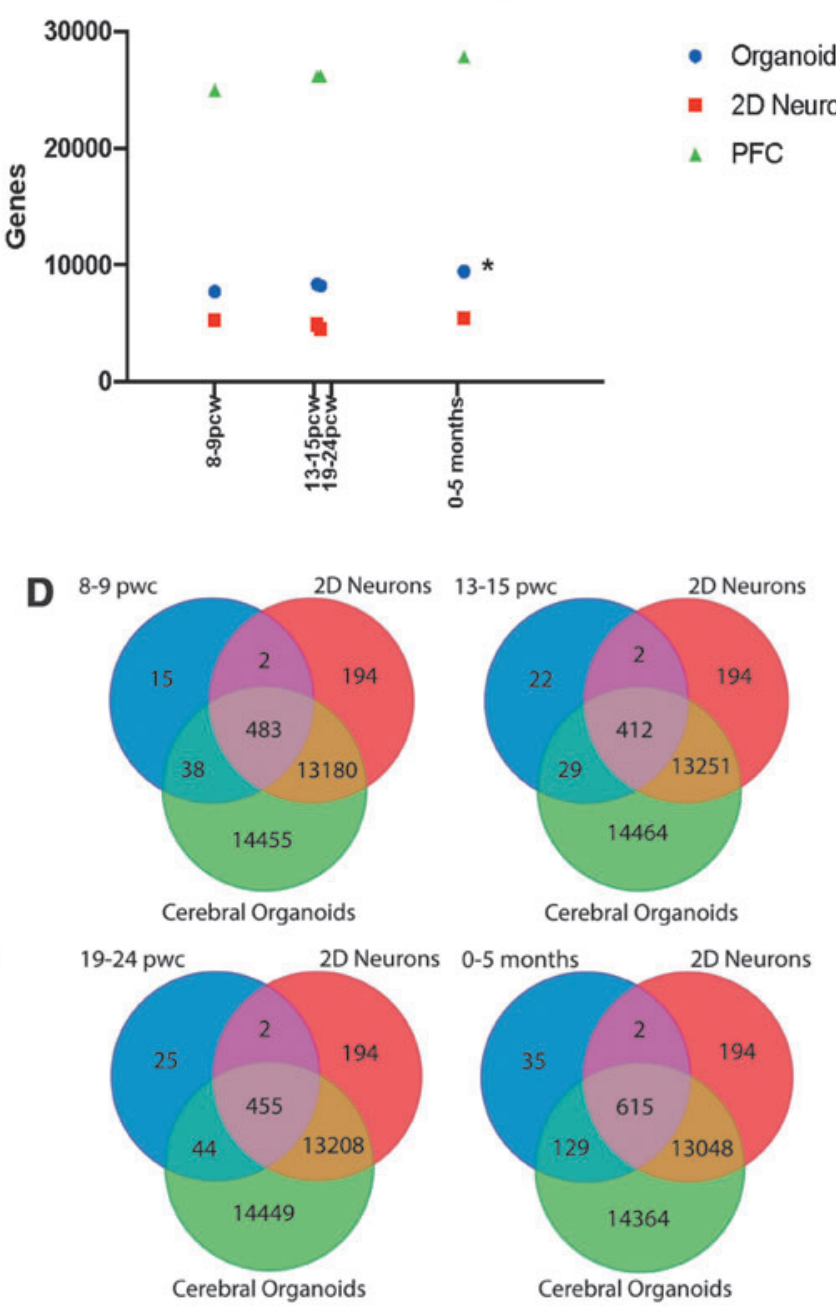

F Correlation Neurological system process

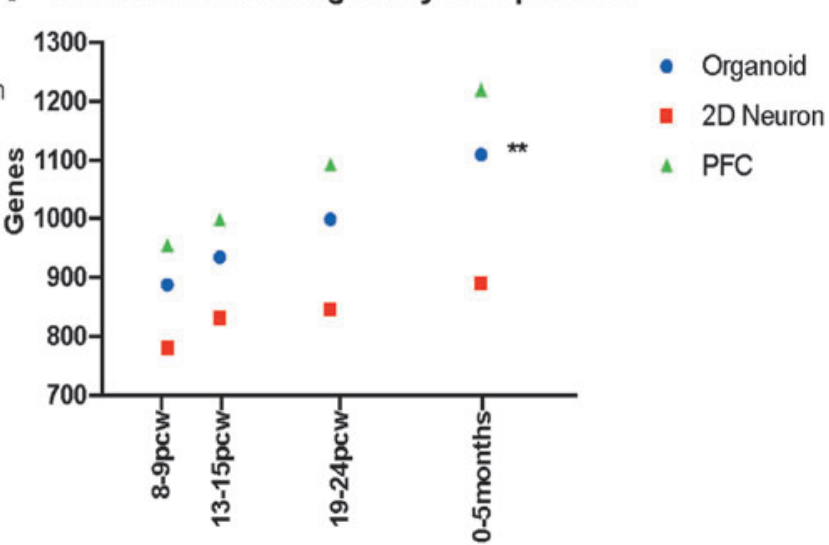

FIG. 4. Comparison of human iPSC-derived cerebral organoid and neuronal culture transcriptome with the transcriptome of the fetal PFC tissue at different stages of development. (A) Overlap of genes expressed in cerebral organoids and cortical neuron cultures with gene expression in fetal PFC at 8-9 pcw, 13-15 pcw, 19-24 pcw, and 0-5 months. (B) Correlation of the transcriptome of fetal PFC, cerebral organoids and cortical neuron cultures. Pearson's correlation coefficient in PFC versus cerebral organoid, $R$ squared $=0.9752 * P$ value $\leq 0.05$. (C) Overlap of nervous system development genes in cerebral organoids and cortical neuron cultures with gene expression in fetal PFC at 8-9 pcw, 13-15 pcw, 19-24 pcw, and 0-5 months. (D) Overlap of neurological system process genes in cerebral organoids and cortical neuron cultures with gene expression in fetal PFC. (E) Correlation between the fetal PFC, cerebral organoid and cortical neuron transcriptomes for the "nervous system development" category. Pearson's correlation coefficient in PFC versus monolayer neurons, $R$ squared $=0.9835 * * P$ value $\leq 0.01$, PFC versus cerebral organoid, $R$ squared $=0.9986 * * * P$ value $\leq 0.001$. (F) Correlation between the fetal PFC, cerebral organoid and cortical neuron transcriptomes for the "neurological system process" category. Pearson's correlation coefficient in PFC versus organoid, $R$ squared $=0.9967, * * P$ value $\leq 0.01$, PFC versus monolayer neurons is not significant. PFC, prefrontal cortex. 
neurons in monolayer cultures to study various aspects of disease biology as well as to study different signaling pathways relevant to disease biology [40-43]. Cerebral organoids present the opportunity to study of live complex neuronal tissue with specific genetic backgrounds $[44,45]$. Monolayer cultures have the predominance of a few cell types and are less heterogeneous while cerebral organoids consist of a higher diversity of neuronal and glial cell types $[46,47]$. A previous study comparing the efficiency of monolayer cultures and three-dimensional methods to generate neurons and astrocytes found that both methods resulted in the generation of similar neural rosettes and gave rise to neurons with similar electrophysiological properties [48]. The three-dimensional methods resulted in higher yield of neural progenitor cells that were positive for PAX6 and NESTIN and resulted in neurons with longer neurites, while the monolayer cultures gave rise to more cells positive for SOX1 [48]. In our earlier studies, we had found that neurons generated from both methods showed spontaneous neuronal firing properties as well as robust responses to chemical and electrical stimuli [22-24].

Cerebral organoids have a larger array of cell types that develop in a three-dimensional structure while twodimensional neuronal cultures have a smaller set of largely neuronal cells that grow within the constraints of a monolayer format [49]. Both of these ex vivo models are being utilized to study fundamental biology as well as diseaserelated cellular processes [50]. However, there has not been a clear understanding of the similarities and differences in the cellular processes and signaling pathways that are active in these model systems. By comparing transcriptomes of the cortical neuron cultures and cerebral organoids generated from the same iPSCs, we highlight the biological processes and pathways operating in the two model systems. The genes that were most significantly enriched in the cerebral organoids include cell adhesion molecules found in more complex tissues, genes expressed in interneurons as well as those involved in plasticity of dendritic spines. These findings are consistent with the greater complexity and cell types that are present in the cerebral organoids [51]. The genes that were most significantly enriched in the monolayer cortical neuron cultures include a calcium-binding protein involved in neuron projection development and cellular stress, a glycoprotein with roles in calcium-dependent protein binding, and signaling pattern recognition receptor activity, as described above.

In analyzing the GO analyses of the DEGs, we found that there was an enrichment of genes involved in tissue development in the cerebral organoids, which is consistent with the more complex structures consisting of multiple cell types that are generated in the development of the cerebral organoids [52]. In the monolayer cortical neuron cultures, we found a relative enrichment of genes in pathways involved in neurogenesis and neuronal differentiation. The monolayer cortical neurons at 3 months consist primarily of excitatory cortical neurons generated from neural progenitor cells [21], which is the likely reason for this relative enrichment in the monolayer cultures vis-à-vis the cerebral organoids. In the GSEA analyses, we were especially intrigued to discover the high correlation with inflammatory response and interferon $-\gamma$ signaling in the cerebral organoids (Fig. 3D-F).
These findings highlight the different biological processes that are robustly represented in these systems, which can serve as a guide in choosing the right model system to study the physiology of human neurobiology as well as to test hypotheses about specific cellular systems that are aberrant in different neuropsychiatric disorders. Our transcriptomic data show that gene expression patterns for nervous system genes in fetal PFC track more closely with iPSC-derived cerebral organoids compared to monolayer cortical neuron cultures. Previous transcriptomic and proteomic experiments with organoids and comparison with fetal brain gene expression profiles had shown that the organoids recapitulate many aspects of cortical development and show enrichment in a number of pathways related to cell-cell adhesion, cytoskeletal organization, and cerebral cortex development [53,54]. Cerebral organoids may especially be suitable for the study of neuroinflammation since microglia develop innately in organoids (Fig. 1C) [55]. We identified microglia in the cerebral organoids by marker analysis and also found enriched expression of microglia-related genes in the cerebral organoids (Fig. 1C and Supplementary Table S4).

Our findings of enriched expression of genes in the interferon- $\gamma$ pathway and immune-related signaling in cerebral organoids, along with the overlap between the enriched interferon- $\gamma$-related genes with gene expression profiles in the fetal PFC transcriptomes, makes them an attractive model to interrogate the biology neuroimmune interactions in health and in disease contexts [56-59]. Two-dimensional neuronal cultures, on the contrary, have robust features related to neurogenesis and neuronal differentiation, and the GO and GSEA analyses indicate enrichment for genes involved in oxidative phosphorylation and cell cycle pathways. Neurons have high energy demands and primarily use glucose through aerobic glycolysis and oxidative phosphorylation for their energy needs, although they will utilize other sources in the setting of nutrient deprivation [60]. There is increased mitochondrial biogenesis and glucose metabolism during neuronal differentiation in two-dimensional human cortical neuron cultures [60]. Cerebral organoids are densely packed, and the core region does not have easy access to nutrients due to the lack of vasculature [61]. This may explain findings of gene expression patterns showing relative enrichment of genes involved in oxidative phosphorylation in the monolayer cortical neuron cultures vis-à-vis the cerebral organoids. Taken together, examination of the transcriptomic profiles presented here adds to our understanding of the relative differences in three-dimensional and two-dimensional cellular model systems that can inform the choice of appropriate model systems to address specific research questions.

\section{Acknowledgment}

We thank Dr. Bruce M. Cohen and Dr. Donna McPhie from Harvard Medical School and McLean Hospital for providing us with the fibroblasts used in the study.

\section{Data Availability Statement}

The datasets generated during the current study have been deposited in NCBI's Gene Expression Omnibus [62] and are accessible through GEO Series accession number GSE140843 (www.ncbi.nlm.nih.gov/geo/query/acc.cgi?acc=GSE140843). 


\section{Author Disclosure Statement}

No competing financial interests exist.

\section{Funding Information}

This work was supported by a National Institute of Mental Health Biobehavioral Research Awards for Innovative New Scientists (BRAINS) Award R01MH113858 (to R.K.), National Institute of Mental Health Clinical Scientist Development Award K08MH086846 (to R.K.), the Doris Duke Charitable Foundation Clinical Scientist Development Award (to R.K.), the Ryan Licht Sang Bipolar Foundation (to R.K.), the Jeanne Marie Lee-Osterhaus Family Foundation NARSAD Young Investigator Award from the Brain \& Behavior Research Foundation (to A.K.), the Phyllis \& Jerome Lyle Rappaport Foundation (to R.K.), the Harvard Stem Cell Institute (to R.K.), and by Steve Willis and Elissa Freud (to R.K.).

\section{Supplementary Material}

Supplementary Figure S1

Supplementary Figure S2

Supplementary Figure S3

Supplementary Table S1

Supplementary Table S2

Supplementary Table S3

Supplementary Table S4

Supplementary Table S5

Supplementary File S1

Supplementary File S2

Supplementary File S3

Supplementary File S4

\section{References}

1. Takahashi K and S Yamanaka. (2015). A developmental framework for induced pluripotency. Development 142: 3274-3285.

2. Lundin A, L Delsing, M Clausen, P Ricchiuto, J Sanchez, A Sabirsh, M Ding, J Synnergren, H Zetterberg, et al. (2018). Human iPS-derived astroglia from a stable neural precursor state show improved functionality compared with conventional astrocytic models. Stem Cell Rep 10:1030-1045.

3. McCaughey-Chapman A and B Connor. (2018). Human cortical neuron generation using cell reprogramming: a review of recent advances. Stem Cells Dev 27:1674-1692.

4. Watmuff B, B Liu and R Karmacharya. (2017). Stem cellderived neurons in the development of targeted treatment for schizophrenia and bipolar disorder. Pharmacogenomics 18:471-479.

5. Hunsberger JG, AG Efthymiou, N Malik, M Behl, IL Mead, X Zeng, A Simeonov and M Rao. (2015). Induced pluripotent stem cell models to enable in vitro models for screening in the central nervous system. Stem Cells Dev 24: 1852-1864.

6. Alsanie WF, OA Bahri, HH Habeeballah, M Alhomrani, MM Almehmadi, K Alsharif, EM Felemban, YS Althobaiti, AH Almalki, et al. (2020). Generating homogenous cortical preplate and deep-layer neurons using a combination of 2D and 3D differentiation cultures. Sci Rep 10:6272.

7. Watmuff B, SS Berkovitch, JH Huang, J Iaconelli, S Toffel and R Karmacharya. (2016). Disease signatures for schizophrenia and bipolar disorder using patient-derived induced pluripotent stem cells. Mol Cell Neurosci 73:96103.

8. O'Shea KS and MG McInnis. (2016). Neurodevelopmental origins of bipolar disorder: iPSC models. Mol Cell Neurosci 73:63-83.

9. Lin M, E Pedrosa, A Hrabovsky, J Chen, BR Puliafito, SR Gilbert, D Zheng and HM Lachman. (2016). Integrative transcriptome network analysis of iPSC-derived neurons from schizophrenia and schizoaffective disorder patients with 22q11.2 deletion. BMC Syst Biol 10:105.

10. Karmacharya R, C Kieling and V Mondelli. (2020). Integrating stem cell-based experiments in clinical research. Eur Psychiatry 63:e62.

11. Donegan JJ and DJ Lodge. (2020). Stem cells for improving the treatment of neurodevelopmental disorders. Stem Cells Dev [Epub ahead of print]; DOI: 10.1089/ scd.2019.0265.

12. Iaconelli J, L Xuan and R Karmacharya. (2019). HDAC6 modulates signaling pathways relevant to synaptic biology and neuronal differentiation in human stem-cell-derived neurons. Int J Mol Sci 20:1605.

13. Renner M, MA Lancaster, S Bian, H Choi, T Ku, A Peer, K Chung and JA Knoblich. (2017). Self-organized developmental patterning and differentiation in cerebral organoids. EMBO J 36:1316-1329.

14. Kelava I and MA Lancaster. (2016). Stem cell models of human brain development. Cell Stem Cell 18:736-748.

15. Jabaudon D and M Lancaster. (2018). Exploring landscapes of brain morphogenesis with organoids. Development 145: dev172049.

16. Qian X, H Song and GL Ming. (2019). Brain organoids: advances, applications and challenges. Development 146: dev166074.

17. Yakoub AM. (2019). Cerebral organoids exhibit mature neurons and astrocytes and recapitulate electrophysiological activity of the human brain. Neural Regen Res 14:757761.

18. Varrault A, L Journot and T Bouschet. (2019). Cerebral cortex generated from pluripotent stem cells to model corticogenesis and rebuild cortical circuits: in vitro veritas? Stem Cells Dev 28:361-369.

19. Trujillo CA, R Gao, PD Negraes, J Gu, J Buchanan, S Preissl, A Wang, W Wu, GG Haddad, et al. (2019). Complex oscillatory waves emerging from cortical organoids model early human brain network development. Cell Stem Cell 25:558-569.e7.

20. Matsui TK, M Matsubayashi, YM Sakaguchi, RK Hayashi, C Zheng, K Sugie, M Hasegawa, T Nakagawa and E Mori. (2018). Six-month cultured cerebral organoids from human ES cells contain matured neural cells. Neurosci Lett 670: $75-82$.

21. Shi Y, P Kirwan and FJ Livesey. (2012). Directed differentiation of human pluripotent stem cells to cerebral cortex neurons and neural networks. Nat Protoc 7:18361846.

22. Kathuria A, K Lopez-Lengowski, B Watmuff, D McPhie, BM Cohen and R Karmacharya. (2019). Synaptic deficits in iPSC-derived cortical interneurons in schizophrenia are mediated by NLGN2 and rescued by $\mathrm{N}$-acetylcysteine. Transl Psychiatry 9:321.

23. Kathuria A, K Lopez-Lengowski, SS Jagtap, D McPhie, RH Perlis, BM Cohen and R Karmacharya. (2020). Transcriptomic landscape and functional characterization of 
induced pluripotent stem cell-derived cerebral organoids in schizophrenia. JAMA Psychiatry 77:745-754.

24. Kathuria A, K Lopez-Lengowski, M Vater, D McPhie, BM Cohen and R Karmacharya. (2020). Transcriptome analysis and functional characterization of cerebral organoids in bipolar disorder. Genome Med 12:34.

25. Lancaster MA and JA Knoblich. (2014). Generation of cerebral organoids from human pluripotent stem cells. Nat Protoc 9:2329-2340.

26. Martin M. (2011). Cutadapt removes adapter sequences from high-throughput sequencing reads. EMBnet 17:10-12.

27. Kim D, G Pertea, C Trapnell, H Pimentel, R Kelley and SL Salzberg. (2013). TopHat2: accurate alignment of transcriptomes in the presence of insertions, deletions and gene fusions. Genome Biol 14:R36.

28. Trapnell C, A Roberts, L Goff, G Pertea, D Kim, DR Kelley, H Pimentel, SL Salzberg, JL Rinn and L Pachter. (2012). Differential gene and transcript expression analysis of RNA-seq experiments with TopHat and Cufflinks. Nat Protoc 7:562-578.

29. Stakkestad Ø, SP Lyngstadaas, B Thiede, J Vondrasek, BS Skålhegg and JE Reseland. (2017). Phosphorylation Modulates Ameloblastin Self-assembly and Ca. Front Physiol 8: 531.

30. Lancaster MA, M Renner, CA Martin, D Wenzel, LS Bicknell, ME Hurles, T Homfray, JM Penninger, AP Jackson and JA Knoblich. (2013). Cerebral organoids model human brain development and microcephaly. Nature 501:373-379.

31. Reinhard JR, A Kriz, M Galic, N Angliker, M Rajalu, KE Vogt and MA Ruegg. (2016). The calcium sensor Copine-6 regulates spine structural plasticity and learning and memory. Nat Commun 7:11613.

32. McKenzie AT, M Wang, ME Hauberg, JF Fullard, A Kozlenkov, A Keenan, YL Hurd, S Dracheva, P Casaccia, P Roussos and B Zhang. (2018). Brain cell type specific gene expression and co-expression network architectures. Sci Rep 8:8868.

33. Iashchishyn IA, MA Gruden, RA Moskalenko, TV Davydova, C Wang, RDE Sewell and LA Morozova-Roche. (2018). Intranasally administered S100A9 amyloids induced cellular stress, amyloid seeding, and behavioral impairment in aged mice. ACS Chem Neurosci 9:1338-1348.

34. Holmskov U, J Mollenhauer, J Madsen, L Vitved, J Gronlund, I Tornoe, A Kliem, KB Reid, A Poustka and K Skjodt. (1999). Cloning of gp-340, a putative opsonin receptor for lung surfactant protein D. Proc Natl Acad Sci U S A 96:10794-10799.

35. Kubo E, N Fatma, P Sharma, T Shinohara, LT Chylack, Y Akagi and DP Singh. (2002). Transactivation of involucrin, a marker of differentiation in keratinocytes, by lens epithelium-derived growth factor (LEDGF). J Mol Biol 320:1053-1063.

36. Gu N, J Wang, Z Di, Z Liu, X Jia, Y Yan, X Chen, Q Zhang and Y Qian. (2019). The effects of intelectin-1 on antioxidant and angiogenesis in HUVECs exposed to oxygen glucose deprivation. Front Neurol 10:383.

37. Maher DM, BK Gupta, S Nagata, M Jaggi and SC Chauhan. (2011). Mucin 13: structure, function, and potential roles in cancer pathogenesis. Mol Cancer Res 9:531-537.

38. Subramanian A, P Tamayo, VK Mootha, S Mukherjee, BL Ebert, MA Gillette, A Paulovich, SL Pomeroy, TR Golub, ES Lander and JP Mesirov. (2005). Gene set enrichment analysis: a knowledge-based approach for interpreting genome-wide expression profiles. Proc Natl Acad Sci U S A 102:15545-15550.

39. Karmacharya R and SJ Haggarty. (2016). Stem cell models of neuropsychiatric disorders. Mol Cell Neurosci 73:1-2.

40. Iaconelli J, JH Huang, SS Berkovitch, S Chattopadhyay, R Mazitschek, SL Schreiber, SJ Haggarty and R Karmacharya. (2015). HDAC6 inhibitors modulate Lys49 acetylation and membrane localization of beta-catenin in human iPSC-derived neuronal cells. ACS Chem Biol 10:883-890.

41. Bame M, MG McInnis and KS O'Shea. (2020). MicroRNA alterations in induced pluripotent stem cell-derived neurons from bipolar disorder patients: pathways involved in neuronal differentiation, axon guidance, and plasticity. Stem Cells Dev [Epub ahead of print]; DOI: 10.1089/ scd.2020.0046.

42. Iaconelli J, J Lalonde, B Watmuff, B Liu, R Mazitschek, SJ Haggarty and R Karmacharya. (2017). Lysine deacetylation by HDAC6 regulates the kinase activity of AKT in human neural progenitor cells. ACS Chem Biol 12:2139-2148.

43. De Los Angeles A and EM Tunbridge. (2020). Unraveling mechanisms of patient-specific NRXN1 mutations in neuropsychiatric diseases using human induced pluripotent stem cells. Stem Cells Dev [Epub ahead of print]; DOI: 10.1089/scd.2020.0017.

44. Srikanth P, VN Lagomarsino, CR Muratore, SC Ryu, A He, WM Taylor, C Zhou, M Arellano and TL Young-Pearse. (2018). Shared effects of DISC1 disruption and elevated WNT signaling in human cerebral organoids. Transl Psychiatry 8:77.

45. Schukking M, HC Miranda, CA Trujillo, PD Negraes and AR Muotri. (2018). Direct generation of human cortical organoids from primary cells. Stem Cells Dev 27:1549-1556.

46. Logan S, T Arzua, SG Canfield, ER Seminary, SL Sison, AD Ebert and X Bai. (2019). Studying human neurological disorders using induced pluripotent stem cells: from 2D monolayer to 3D organoid and blood brain barrier models. Compr Physiol 9:565-611.

47. O'Connell L and DC Winter. (2020). Organoids: past learning and future directions. Stem Cells Dev 29:281-289.

48. Chandrasekaran A, HX Avci, A Ochalek, LN Rösingh, K Molnár, L László, T Bellák, A Téglási, K Pesti, et al. (2017). Comparison of 2D and 3D neural induction methods for the generation of neural progenitor cells from human induced pluripotent stem cells. Stem Cell Res 25: 139-151.

49. Liu C, A Oikonomopoulos, N Sayed and JC Wu. (2018). Modeling human diseases with induced pluripotent stem cells: from 2D to 3D and beyond. Development 145: dev. 156166.

50. Oliveira B, A Çerağ Yahya and G Novarino. (2019). Modeling cell-cell interactions in the brain using cerebral organoids. Brain Res 1724:146458.

51. Christian KM, H Song and GL Ming. (2020). Using twoand three-dimensional human iPSC culture systems to model psychiatric disorders. Adv Neurobiol 25:237-257.

52. Costamagna G, L Andreoli, S Corti and I Faravelli. (2019). iPSCs-Based Neural 3D Systems: a multidimensional approach for disease modeling and drug discovery. Cells 8: 1438.

53. Camp JG, F Badsha, M Florio, S Kanton, T Gerber, M Wilsch-Bräuninger, E Lewitus, A Sykes, W Hevers, et al. (2015). Human cerebral organoids recapitulate gene expression programs of fetal neocortex development. Proc Natl Acad Sci U S A 112:15672-15677. 
54. Nascimento JM, VM Saia-Cereda, RC Sartore, RM da Costa, CS Schitine, HR Freitas, M Murgu, RA de Melo Reis, SK Rehen and D Martins-de-Souza. (2019). Human cerebral organoids and fetal brain tissue share proteomic similarities. Front Cell Dev Biol 7:303.

55. Ormel PR, R Vieira de Sá, EJ van Bodegraven, H Karst, O Harschnitz, MAM Sneeboer, LE Johansen, RE van Dijk, N Scheefhals, et al. (2018). Microglia innately develop within cerebral organoids. Nat Commun 9:4167.

56. Chukwurah E, A Osmundsen, SW Davis and SB Lizarraga. (2019). All Together Now: modeling the interaction of neural with non-neural systems using organoid models. Front Neurosci 13:582.

57. Haenseler W and L Rajendran. (2019). Concise Review: modeling neurodegenerative diseases with human pluripotent stem cell-derived microglia. Stem Cells 37: 724-730.

58. Winkler CW, TA Woods, BR Groveman, AB Carmody, EE Speranza, CA Martens, SM Best, CL Haigh and KE Peterson. (2019). Neuronal maturation reduces the type I IFN response to orthobunyavirus infection and leads to increased apoptosis of human neurons. J Neuroinflammation $16: 229$.

59. Mondelli V, AC Vernon, F Turkheimer, P Dazzan and CM Pariante. (2017). Brain microglia in psychiatric disorders. Lancet Psychiatry 4:563-572.
60. Agostini M, F Romeo, S Inoue, MV Niklison-Chirou, AJ Elia, D Dinsdale, N Morone, RA Knight, TW Mak and G Melino. (2016). Metabolic reprogramming during neuronal differentiation. Cell Death Differ 23:1502-1514.

61. Vargas-Valderrama A, A Messina, MT Mitjavila-Garcia and H Guenou. (2020). The endothelium, a key actor in organ development and hPSC-derived organoid vascularization. J Biomed Sci 27:67.

62. Edgar R, M Domrachev and AE Lash. (2002). Gene Expression Omnibus: NCBI gene expression and hybridization array data repository. Nucleic Acids Res 30:207-210.

Address correspondence to: Prof. Rakesh Karmacharya Center for Genomic Medicine Massachusetts General Hospital 185 Cambridge Street, CPZN6 Boston, MA 02114

USA

E-mail: karmacharya@mgh.harvard.edu

Received for publication April 7, 2020

Accepted after revision August 28, 2020

Prepublished on Liebert Instant Online August 29, 2020 